\title{
The status of the Ferruginous Duck Aythya nyroca in Turkey
}

\author{
GUY M. KIRWAN
}

\section{Summary}

Both breeding and midwinter populations of the Ferruginous Duck Aythya nyroca are in decline throughout Europe. As part of an action plan for the species currently being prepared, a comprehensive Turkish site inventory was made. The Turkish breeding and midwinter populations (500-600 pairs and $0-1,500$ birds respectively) are amongst the most important in Europe but have been subject to significant and probably long-term declines. Of 25 breeding sites identified in this study, only 10 are considered to hold more than to pairs, seven are probably no longer suitable and the rest probably all support fewer than five pairs. Thirty-one localities have held birds in winter but of these 18 have been unoccupied in the 1990s. Significant numbers appear to remain in Turkey only during particularly mild winters. Measures for its effective conservation in Turkey are outlined. A comprehensive site inventory, based on fieldwork directed to potential breeding localities, and monitoring of important sites identified by this study and earlier surveys are urgently required.

\section{Introduction}

Formerly regarded as one of the most common ducks in Europe during the first quarter of the twentieth century, the Ferruginous Duck Aythya nyroca has subsequently undergone a large-scale and, in some areas, very dramatic decline, although the European population is still considered to comprise $50 \%$ of the total world population (A. D. Callaghan, unpublished). The Ferruginous Duck is currently considered Vulnerable (Collar et al. 1994). Its status in Europe has been reviewed by Tucker and Heath (1994). Between 1970 and 1990, 15 countries recorded declines in breeding population, most marked in the Dniestr-Dneipr region of Ukraine and Moldova, where there has been a 90\% decrease since the 1960s. Four very small (all less than 100 pairs) and two significant (Croatia, 200300 pairs and Hungary, 1,200-1,600 pairs) populations are stable or increasing. A. D. Callaghan (unpublished data) considers the European breeding population to be between 17,000 and 24,000 pairs, excluding unknown numbers in Armenia, Bosnia-Herzegovina and Georgia, while the continent's winter population has declined markedly. Tucker and Heath (1994) suggested a Turkish population in the region of 1,000-3,00o pairs, its trends unknown. Kasparek (1992), in the most recent comprehensive review of Turkish bird status and distribution, described the species as "Very local, but not rare breeding bird in all regions of Turkey with the exception of the South-east. Appears in moderate numbers on passage throughout the country, principally in western regions. Also a winter visitor in much smaller numbers. The January count is usually below 100 birds". 


\section{Methods}

As part of the development of an action plan for the conservation of the Ferruginous Duck, a comprehensive review of Turkish records was undertaken. Records were collated from Turkey Bird Reports (1966-91) and their source material to date (1996), the published literature (where this was easily accessible) and from unpublished information on a database held in Turkey (DHKD). Records are presented according to the regional subdivisions of Turkey as employed in the Turkey Bird Report (see Beaman 1986 and Martins 1989). Some data in the earlier Turkey Bird Reports (1966-69) are rather vague, listing small counts from a group of sites e.g. "remainder of Central Plateau". Such counts are always taken to refer to one site in the totals and discussion below.

\section{Results and discussion}

Data from 92 localities, of which 25 are listed as breeding localities (some with little evidence), are presented. Several other sites (c. 10) probably support breeding birds but no proof of breeding is available in either the published or unpublished literature. Most breeding localities support relatively small populations. Only 10 areas are currently considered to hold over 10 pairs: Kizilirmak Delta (Black Sea Coastlands) 150-200 pairs (Hustings and van Dijk 1994), Kocaçay Delta (Western Anatolia) 70-100 pairs (Ertan 1996), Göksu Delta (Southern Coastlands) 30 pairs (A. J. Green unpublished, Magnin and Yarar 1997), Sultansazligi (Central Plateau) 20 pairs (Magnin and Yarar 1997), Mogan Gölü (Central Plateau) 20 pairs (O. Görgün in litt. to DHKD 1994), Marmara Gölü (Western Anatolia) 15 pairs (G. Eken in litt. to DHKD), Eregli marshes (Central Plateau) 10 pairs (Magnin and Yarar 1997), Aksehir Gölü (Central Plateau) 10 pairs (Langeveld and Ruiters 1994), Karamik Gölü (Southern Coastlands) 1o pairs (Langeveld and Ruiters 1994) and Kulu Gölü (Central Plateau) 1o pairs (Magnin and Yarar 1997).

Of the other 14 known breeding localities, three (Abant Gölü, Emir Gölü and Ilgaz Gölü) are listed by Kumerloeve (1961) without details and probably all are no longer suitable. Two others (Amik Gölü and Gogenç Gölü) have been drained or otherwise destroyed, two (Van marshes and Edremit marshes) have been largely inundated due to rising water levels on Van Gölü, and the other seven all probably support less than five pairs. In addition several important breeding localities, e.g. Eregli marshes and suspected breeding areas, e.g. Yarma marshes, are either subject to on-going drainage schemes or have already been drained. Full details of such developments are presented in Magnin and Yarar (1997). It appears unlikely on current evidence that the Turkish breeding population exceeds 500 pairs, significantly less than the minimum 1,000 pairs suggested by Tucker and Heath (1994). Further work, presently hampered by the adverse political situation in eastern Anatolia, is urgently required as several areas listed here, from which breeding data are lacking, may support breeding birds. However, it is unlikely that any of these hold populations in excess of 10-20 pairs.

Of the total number of localities listed, 33 have held birds in winter (November-February), of which 10 are winter-only sites. There is strong evidence 


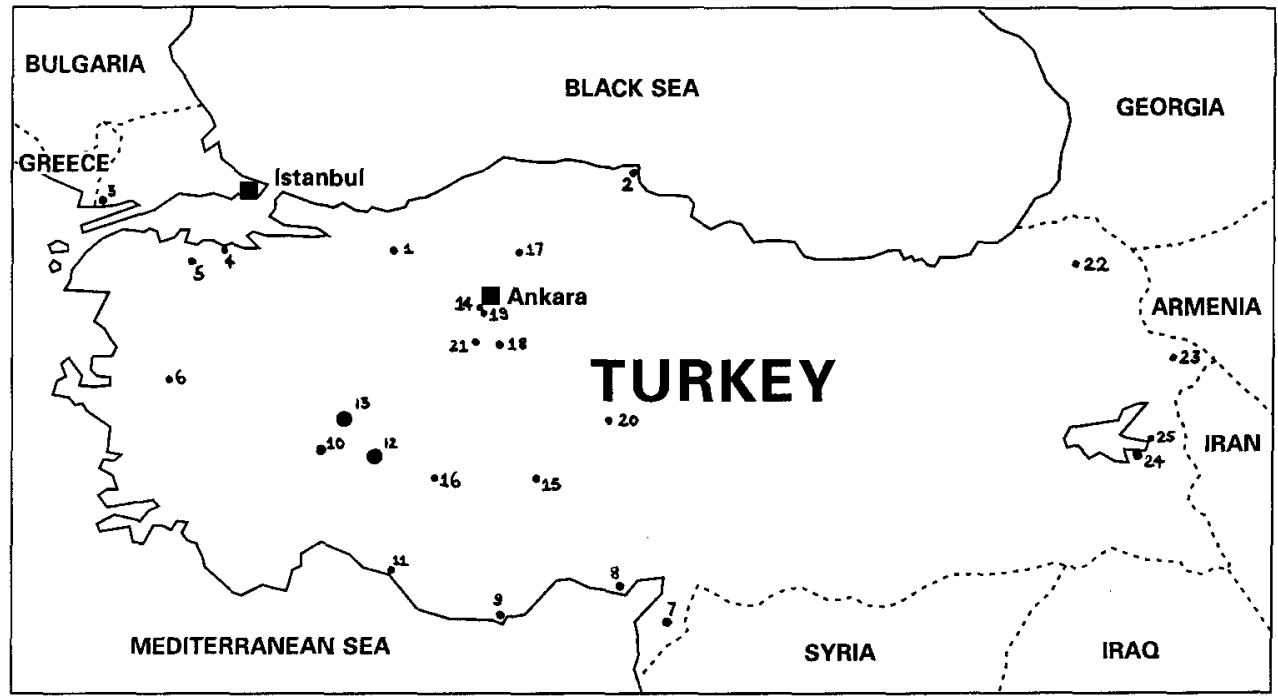

Figure 1. Turkey, showing position of the breeding localities mentioned in the text. 1, Abant Gölü; 2, Kizilirmak Delta; 3, Meriç Delta; 4, Kocaçay Delta; 5, Manyas Gölü; 6, Marmara Gölü; 7, Amik Gölü; 8, Çukurova Deltas; 9, Göksu Delta; 10, Karamik Gölü; 11, Manavgat-Karpuz river area. 12, Aksehir Gölü; 13, Eber Gölü; 14, Emir Gölü; 15, Eregli marshes; 16, Gogenç Gölü; 17, Ilgaz Gölü; 18, Kulu Gölü; 19, Mogan Gölü; 20, Sultan marshes; 21, Uyuz Gölü; 22, Ardahan marshes; 23, Dogubeyazit marshes; 24, Edremit marshes; 25, Van town marshes.

of a decline in recent winters. Of the total number of sites with winter records, 18 have not been occupied in the 1990s. DHKD have operated increasingly extensive winter counts, with full censuses attempted in 1990, 1992, 1993 and 1996, designed to provide an accurate overview of the numbers of waterfowl utilizing Turkish wetlands at this season. A further six localities held birds only in the exceptional winter of 1990, when a total of 1,329 were recorded at eight sites. In contrast the 1996 count, involving three teams totalling 29 individuals and visiting 67 wetlands, encountered no Ferruginous Ducks, despite recording the second highest waterfowl total of $1,879,068$ birds (DHKD 1996), compared with 500,000-700,000 birds counted during winter in the last decade. Furthermore, only four sites held birds in more than one winter in the 1990s, despite generally improved counting techniques, more observers in the field at this season and increased coverage with more wetlands being visited.

\section{Summary of the records}

A regional summary of the most important sites in Turkey for Ferruginous Duck is presented below. Full details of all records located by the author are in Appendix 1.

Black Sea Coastlands Two breeding localities (Abant Gölü and Kizilirmak Delta) and five other localities, of which two have records from the breeding season. 
Thrace One breeding site (Meriç Delta) and five other localities, of which two have records from the breeding season.

Western Anatolia Three breeding sites (Kocaçay Delta, Manyas Gölü and Marmara Gölü) and seven other localities, of which three have records from the breeding season.

Southern Coastlands Five breeding sites (Amik Gölü, Çukurova Deltas, Göksu Delta, Karamik Gölü and the Manavgat-Karpuz river area) and 15 other localities, of which five have breeding season records. One breeding site, Amik Gölü, has now been drained and at least two other sites from which there are breeding season records have also been lost to development.

Central Plateau Ten breeding sites (Aksehir Gölü, Eber Gölü, Emir Gölü, Eregli marshes, Gogenç Gölü, Ilgaz Gölü, Kulu Gölü, Mogan Gölü, Sultan marshes and Uyuz Gölü) and 19 other localites, of which eight have breeding season records. Three breeding localities (Emir Gölü, Gögenç Gölü and Ilgaz Gölü) are either no longer suitable for nesting or have been drained, and of the other localites with breeding season records, three have also been drained.

South-east No breeding localities identified. One of two other sites has a breeding season record but this may no longer be suitable.

East Four breeding sites (Ardahan marshes, Dogubeyazit marshes, Edremit and Van town marshes) and 17 other localities, of which 13 have breeding season records. Both Edremit and Van town marshes have been inundated by rising water levels on Van Gölü and are probably no longer suitable, whilst of the other sites with breeding season records, approximately four have suffered similar fates.

\section{Conservation prospects}

The Turkish population of Ferruginous Duck, although considerably smaller than predicted by Tucker and Heath (1994), is still significant in European and global terms. Only five other breeding populations reviewed by A. D. Callaghan (unpublished) certainly exceed it: Croatia (1,000-3,000 pairs), Hungary (1,2001,600 pairs), Moldova (1,000-1,300 pairs), European Russia (500-1,500 pairs) and Ukraine (3,500-5,00o pairs). Despite the irregularity of the species's winter occurrence in Turkey, just five other European states possess significant midwinter populations: Azerbaijan (3,000-5,000 birds), Croatia (o-200 birds), Italy, including Sardinia (100-400 birds), Romania (100-200 birds) and Ukraine (1,500 birds). The Turkish midwinter population has shown a decrease of between $20 \%$ and $49 \%$ since 1976 and is presently considered to be between $0-1,500$ birds.

The Ferruginous Duck was recently declared a protected species in Turkey (G. Eken in litt.). The first step towards its effective conservation must be the implementation of full legal protection. A. D. Callaghan (unpublished action plan) lists a number of other key objectives for conservationists concerned with 
this species in Turkey, chief of which are: to develop a national strategy for the species's conservation and its habitat; to promote the adequate protection and management of key sites identified in the course of this study; to restore, where possible, key sites which have been drained; to monitor and prevent hunting mortality and activities; to prepare a comprehensive site inventory and assessment of the conservation status of each wetland; and to develop regular censusing of all important sites for the species at appropriate seasons.

\section{Acknowledgements}

I am grateful to my colleagues at DHKD, Istanbul, particularly Gernant Magnin and Murat Yarar, who provided many records for this summary including details from Magnin and Yarar (1997) prior to publication, as well as their good friendship over the last five years; Dr Janet Hunter and Des Callaghan at the Wildfowl and Wetlands Trust (Slimbridge) encouraged this review and provided some of the records whilst G. Eken provided much useful discussion of the species' status. Katherine Robinson provided some details of the Anatolian Duck Project Oxford University Expedition 1996. All those observers who submitted records to the Turkey Bird Report are acknowledged for their important contribution to this paper.

\section{Appendix 1. The records}

All records are from the relevant Turkey Bird Report, unless otherwise stated. Sites mentioned are in Figure 1 or on the Official Road Map of Turkey (Kartographischer Verlag Reinhard Ryborsch 1994).

\section{Black Sea Coastlands ( 2 breeding localities; 5 other sites) \\ Main sites (including all breeding localities)}

Abant Gölü: listed as a breeding bird without details (Kumerloeve 1961, Kumerloeve 1970).

Kizilirmak Delta: $150-200$ breeding pairs estimated in 1992 when recorded in most marshland and some grassland plots with pairs present from 17 March onwards; breeding was proven on several occasions, two examples given below (Hustings and van Dijk 1994); up to 30, 14-16 July 1966, 3, 28 September 1967 (Vielliard 1968); 61, winter 1969-70; 11, 4 August 1967; 5, 8-9 June 1969; 61, 26 January 1970; 2-3, 30 April 1970; 4 pairs, 2 August 1971; female with 7 juveniles, 6 August 1971; 22, 22 January 1972; 15, 10 September 1973; recorded, 25 May 1984 (Heunk unpubl.); "many", 15 July 1986 (Webb unpubl.); 26, 11 June 1987 (Hirschfeld unpubl.); 35, 26 June 1987 (Eames unpubl.); up to 6, 17-18 July 1987 (Vermeulen unpub.); 30, 30 June 1989 (Young unpubl.); 30, 17 June 1990 (Forsten unpubl.); 30 , 6 February 1991 (Özesmi unpubl.); 14, 9-12 May 1991 (Vos unpubl.); 1, 10 June 1991 (Kirwan unpubl.); 7, 27 July 1991 (Özesmi unpubl.); 1, 11 August 1991 (Özesmi unpubl.); 2, 18 August 1991 (Özesmi unpubl.); nest with 6 eggs, 5 May 1992 (Hustings and van Dijk 1994); 2 females with 3 and 4 fledglings respectively; 1 June 1992 (Hustings and van Dijk 1994); 29, 7 June 1992 (Hustings and van Dijk 1994); 4, 27 March 1993 (DHKD database); 1, 29 May 1993 (Kirwan unpubl.); 22, 14 July 1993 (Hogg unpubl.); 30, 1 October 1994 (DHKD database); 20, 7 May 1995 (DHKD database); 10, 24-26 September 1995 (Kirwan unpubl.); 14, 25 October 1995 (DHKD database).

Yeniçaga Gölü: 5, 11 April 1965 (Kiliç and Kasparek 1987); 1, 27 May 1971; four records of singles in September, no year(s) given (Kiliç and Kasparek 1987); 1, 30 September 1994 (DHKD database).

Yesilirmak Delta: up to 30, 14-16 July 1967.

\section{Other sites}

Recorded, Lâdik Gölï, 21 May 1984 (Heunk unpubl.).

2, on sea between Trabzon and Ordu, late May 1968.

3, Yedikir Baraji, 24 September 1994 (DHKD database), 4, 20 January 1997 (G. and H. Welch). 


\section{Thrace (1 breeding site; 4 other localities)}

Main sites (including all breeding localities)

Meriç Delta: on the Greek side of the delta (Evros) regarded as a scarce breeding bird, regularly seen in small numbers (Bauer and Müller 1969); 10, winter 1968-69; 27, 15 November 1970 (regularly recorded in smaller numbers November/January in 1970-73); at least 7 pairs in suitable breeding habitat, 23-24 June 1973; 1, I June 1994 (DHKD database).

\section{Other sites}

Considered rare at the Bosphorus between 1888-1894 (Mathey-Dupraz 1924).

Büyükçekmece: "suspected to breed" (Magnin and Yarar 1997); 4, 7 October 1977 (DHKD database); 1, 18 September 1987 (Ullman unpubl.); 1, 17 December 1994; 2, 15 September 1995 (DHKD database). 3, "near the Greek border", 17 April 1966.

Terkos Gölü: 5, 31 January 1995; 2, 18 March 1995 (both records DHKD database).

\section{Western Anatolia ( 3 breeding sites; 7 other localities) \\ Main sites (including all breeding localities)}

Bafa Gölü: 8, 15 September 1982 (Kasparek 1988); pair, 28 March 1984 (Kasparek 1988); 6, 13 October 1988 (Mortemore unpubl.); 32, 22 January 1990 (DHKD database).

Kocaçay Delta: population estimated at 70-100 pairs in 1993 (Ertan 1996); 1-10, 19 September 1987 (DHKD database); 4, 20 May 1993 (DHKD database); 10+, 7 April 1996 (Whitehouse unpubl.).

Manyas Gölü: adults with young, undated either 1966 or 1967 and listed as breeding by Kiziroglu and Kiziroglu (1987); c. 3,00o, 21 January 1967; up to 3, mid-April and early May 1968; up to 6, 4 August-30 September 1968; 5, 29 August-2 September 1971; 3, 25 May 1995 (DHKD database).

Marmara Gölü: pair, 1o May 1973; 20-25 pairs estimated 1996 (G. Eken in litt. to DHKD); 860, 25 January 1990; 20, 12 May 1993; 5, 21 March 1995; 18, 4 June 1995 (all records DHKD database).

Uluabat (Apolyont) Gölü: 20, winter 1968-69; up to 3, mid-April and early May 1968; up to 6, 4 August-30 September 1968; 18, 8 September 1970; at least 40, 7 September 1971; 2, 6 August 1972; 1, 22 August 1972; pair, 28 June 1973; 65, 18 August 1987 (Gregory unpubl.); 16, 22 September 1987 (Ullman unpubl.); 3-5, 24-25 May 1990 (Ullman unpubl.); 13, 23 September 1992 (DHKD database); 10, I8 March 1993 (DHKD database); 5, 20 May 1993 (DHKD database); 3, 26 May 1995 (DHKD database); 8, 15 September 1995 (Kirwan unpubl.).

\section{Other sites}

10, Dalyan estuary, 29 March 1987 (Kiliç and Kasparek 1989).

10, marsh near Erdek, I4 April 1970.

Isikli Gölü: 300, winter 1968-69; 1, 17 January 1971; 5, 22 January 1989 (DHKD database); 12, 18 January 1990 (DHKD database); 97, 29 January 1992 (DHKD 1992b).

1, Iznik Gölü, 28 October 1967 (Vielliard 1968).

2, Karakuyu marshes, 28 March 1995 (DHKD database).

Küçük Menderes Delta: 2, 22 March 1995 (DHKD database); 1, 15 May 1995 (Kane unpubl.); pair, 11 April 1996 (Whitehouse unpubl.).

1, Sapanca Gölü, 4 June 1989 (Morton et al. unpubl.).

\section{Southern Coastlands ( 5 breeding sites; 15 other localities)}

Main sites (including all breeding localities)

Amik Gölü: Meinertzhagen shot a female with an egg in the oviduct on 27 May 1933; 120-150, 8 May 1953; nest with eggs, 2 I May 1953; adult with 5 young, 3 September 1956; breeding in May 1962 with at least 20, 9 May 1962 (all records, Kumerloeve 1963); flock, 24 April 1965 (Kumerloeve 1966); "few", 23 January 1967; 50, 14-16 July 1967 (Vielliard 1968); 1, 3-7 September 1967 (Vielliard 1968); 300, January 1968; 15, 1 May 1970.

Beysehir Gölü: 5, 15 May 1964 (Vauk 1973); c. 80, 7-8 February 1967; 10-25, 1-10 May 1967; recorded, May 1968; 4, 20 December 1970; 10, 22 May 1982 (Berg unpubl.); 6, 14 January 1990 (Dijksen unpubl.). Burdur Gölü: 1, 26 June 1964 (Kumerloeve 1970); 1, 18 July 1965 (Kumerlove 1970); 1, 6 October 1970; 1, 3 September 1972; at least 65, 16 September 1972; 1, 16-17 January 1973; 11, 31 August 1987 (Lister unpubl.); 10, 16 January 1990; 2, 27 October 1994; 1, 23 July 1995 (all 199os records DHKD database). 
Çukurova: 2 breeding pairs and nest with eight eggs, Aynaz Gölü, 15 May 1962 (Kumerlove 1970); considered a breeding bird with observations on Agyatan Gölü, at the mouth of River Tarsus and River Ceyhan, 6 May 1964 (Warncke 1965); recorded, south of Tarsus, 8 April 1967 (Kumerloeve 1970); 4, Akyatan Gölü, 27 November 1970; 3, Tuzla Gölü, 4 April 1982 (Gyssel unpubl.); 4, Ceyhan River, 31 March 1985 (Novatours unpubl.); 1, west of Agyatan Gölü, 16 May 1987; 2, Omer Gölü, 18 May 1987 (both 1987 records, van der Have et al. 1988); 1, Akyatan Gölü, 12 March 1990; 1 Tuzla Gölü, 21 March 1990; 8, Tuzla Gölü, 22 March 1990; drake, Tuzla Gölü, 23 March 1990; 2, Küçük Karatas, 29 March 1990; pair Küçük Karatas, 7-12 May 1990 (all 1990 records, Kivit et al. 1994); 3, Akyatan Gölü, 29 January 1992 (DHKD 1992b); 1, Yumurtalik, 28 January 1992 (DHKD 1992b); 7, Karatas, 30 September 1994 (Thorpe unpubl.); 2, Yesikoy lake, 7 October 1994 (Thorpe unpubl.); 1, Karatas, 26 March 1995 (DHKD database).

Göksu Delta: listed as a migrant breeder and winter visitor (DHKD 1992a), with up to 30 pairs estimated to breed (A. Green in litt. to DHKD); c. 10, 10-11 July 1967 (Vielliard 1968); 13, 30 April 1970; 20, 9-13 October 1970; "some", late September 1971; at least 3, 21-25 September 1973; at least 20, 26 September 1974; 50, 21-22 May 1975; 4, 24 May 1975; 3, 29 June 1984 (Webb unpubl.); 1, 13 May 1985 (Grace unpubl.); 4, 19 May 1986 (Warren unpubl.); 10, 5 July 1986 (Webb unpubl.); 1, 19 May 1987 (Eames unpul.); drake, 27 May 1987 (Hirschfeld unpubl.); 18, 28-29 August 1987 (Lister unpubl.); up to 13, 27-28 April 1988 (Rushforth unpubl.); up to 11, 12-13 May 1988 (Ullman unpubl.); 1, 25 May 1988 (Hodgson unpubl.); up to 30, 1-2 August 1988 (de Bruin unpubl.); up to 8, 28-30 August 1988 (Moore unpubl.); 20, 11 September 1988 (Huhta-Koivisto unpubl.); at least 10, 25-26 September 1988 (Green unpubl.); 8, 23 February 1989 (DHKD database); up to 20, 11-13 May 1989 (Ullman unpubl.) up to 8, 17-18 May 1989 (Young unpubl.); 1, 27 May 1989 (Green unpubl.); 10, 8 June 1989 (Smethurst unpubl.); 1, 15 June 1989 (Jonassohn unpubl.); 2, 27 February 1990 (DHKD database); 15, 12 April 1990 (Lundberg unpubl.); at least 50, 30 June 1990 (Kirwan unpubl.); adult with 3 young, 11 July 1991 (Littlewood unpubl.); 2, 9 September 1991 (Kirwan unpubl.); 4, 31 January 1992 (DHKD 1992b); 36, 4 May 1992 (Petersson unpubl.); 8, 22 March 1993 (van der Winden unpubl.); 5, 16 April 1993 (Smith unpubl.); 7, 14 May 1993 (Kirwan unpubl.); 8, 15 May 1993 (Kirwan unpubl.); 2, 11 June 1994 (Rosier unpubl.); 5, 23-25 January 1995 (van den Berk unpubl.); 2-3 pairs, 11-12 April 1995 (Muddeman unpubl.); 6, 14 April 1996 (Royle unpubl.); 3, 22 April 1996 (Royle unpubl.); 10, 23 April 1996 (Whitehouse unpubl.).

Karamik Gölü: 1o pairs estimated to breed (Langeveld and Ruiters 1994); 1, 31 January 1967; 10-25, 1-10 May 1967; 85, winter 1968-69; 50, winter 1969-70; up to 90, 12 July 1968, up to 90, 25 August 1968, 50, 19 January 1970; 11, 14 December 1970; at least 1, 9 August 1971; at least 2, 18-19 July 1972; at least 18, 12 April 1973; c. 10, 2 June 1975; 8, 24 May 1982 (Berg unpubl.); 1, 24 September 1984 (Kasparek unpubl.); female with 8 young, 4 July 1989 (Richardson unpubl.); 20 mainly females, with young, 30 June 1994 (Langeveld and Ruiters 1994); 1, 28 May 1995 (DHKD database); 6, 16 September 1995 (Kirwan unpubl.); less than 10, 17 July 1996 (Anatolian Duck Project 1996).

Lakes in western Taurus (other than Karamik and Beysehir Gölï): 7, January 1967; 6, January 1968; 20, winter 1968-69; 40, winter 1969-70.

Manavgat-Karpuz river area: breeding bird (Erdogan and Turan unpubl.) with observations in January, March, July, October and November and a maximum of 4 birds between September 1992-August 1995.

\section{Other sites}

1, Alanya, 3 October 1974 .

10o, Corak Gölï, 14 January 1970.

Recorded, near Elmali, 11 June (no year) (Kumerloeve 1961).

Drake, Erdemli, 24-31 March 1972.

20, near Finike, 5 September 1974.

Female, $16 \mathrm{~km}$ west of Finike, 4 September 1986 (Counsell unpubl.).

Gavur Gölü: 1, 18 July 1967 (Vielliard 1968); 3, 24 March 1994 (DHKD database); 2, 22 April 1996 (DHKD database).

4, Hoyran Gölü, 17-18 January 1970.

At least 2, Karagöl, 29 August 1970.

400, Salda Gölï, 19 January 1990 (DHKD database).

4, Sugla Gölü, I9 May 1982 (Berg unpubl.).

7, Yarisli Gölï, 19 January 1990 (DHKD database). 


\section{Central Plateau (10 breeding sites; 19 other localities)}

Main sites (including all breeding localities)

General: Flocks of up to 30 but usually fewer than to seen irregularly on passage in this region in 1966 and 1967 between 20 February (1966) and mid-May and late July to October (latest 20 October 1967), with some remaining until November (latest 18th) in 1968-69.

Aksehir Gölü: 1o pairs estimated to breed (Langeveld and Ruiters 1994); c. 100, 19-29 May 1967; up to 8, May 1968 and 1969; 10, 13-14 December 1970; c. 15, 4-8 August 1971; 3, 28 November 1971; 1, 29 August 1974; 8, 29 August 1975 (Max Kasparek database); 2, 12 July 1977 (Meininger unpubl.); 1, 13 July 1977 (Meininger unpubl.); 15, 19 June 1994 (Langeveld and Ruiters 1994).

Balikdami: probably bred, 1966 or 1967; 5 pairs, 4 May 1969; 1, 5 January 1970; 90, 25 December 1970; 2, 2 May 1992; 3, 26 June 1993; 6, 5 March 1994; 2, 7 May 1994; 1, 11 September 1994; 9, 30 May 1995; 6, 16 April 1996 (all 1990s records DHKD database).

Çavusçu Gölü: 2, 28 November 1971; 2, 13 April 1973; 6, 28 August 1974; 5, 22 March 1993 (DHKD database); 25, 17 September 1995 (Kirwan unpubl.).

Eber Gölï: at least 30, 19-29 May 1966; up to 8, May 1968 and 1969; 5, 4 May 1971; 30, 8 August 1971; 70, 15 December 1970; drake, 20 April 1988 (van Winden et al. 1989); 3 (female with young), 20 June 1994 (Langeveld and Ruiters 1994); 4, 16 September 1995 (Kirwan unpubl.). Total of 60 on Eber, Aksehir and Çavusçu Gölü, January 1968. Lakes on Central Plateau other than latter three lakes: 18, January 1968; 19, winter 1969-70.

Emir Göliui: listed as a breeding bird without details (Kumerloeve 1961); 8, 21 March 1969.

Eregli marshes: current breeding population considered to be 10 pairs (Magnin and Yarar 1997); 19, 5 January 1970; 5 pairs, 8 June 1971; 4, 21 November 1971; 1, 28 December 1982 (Kiliç and Kasparek 1990); 2, 25 April 1983 (Weitkowitz unpubl.); 2, 28 December 1983 (Kiliç and Kasparek 1990); 30, 27 September 1984 (Kasparek unpubl.); recorded, 19 July 1986 (Webb unpubl.); 150, 28 June 1987 (Eames unpubl.); 6, 22-24 May 1988 (Hodgson unpubl.); 10, 11 May 1989 (Green unpubl.); 20, 19 May 1989 (Young unpubl.); c. 10, 24 May 1989 (Clarke unpubl.); at least 10, 28-29 May 1989 (Green unpubl.); 20, 24 June 1989 (Smethurst unpubl.); at least 150, 21 June 1989 (Richardson unpubl.); 2, 9 March 1990 (DHKD database); 2, 17 June 1990 (Kirwan 1990); 60, 21 June 1990 (Forsten unpubl.); 1, 23 June 1990 (DHKD database); 11, 1 June 1991 (Kirwan unpubl.); 6, 7 June 1991 (Bradshaw unpubl.); 8, 30-31 March 1993 (van der Winden unpubl.); 20, 3 July 1993 (Hogg unpubl.); 2, 31 May 1994 (Rosier unpubl.); "few", I June 1994 (Smith unpubl.); 3-5 pairs, 20 April 1995 (Muddeman unpubl.); 2, 29 April 1996 (DHKD database); pair, 4 May 1996 (Whitehouse unpubl.), less than 10, 24 July 1996 (Anatolian Duck Project 1996).

Gogenç Gölü: 5 pairs, 2 June 1972; 5 females each with juveniles, 6 August 1972.

Hotamis marshes (including Süleymanhaci Gölü): 1, 7 January 1971; 3 pairs, 6-7 June 1971; 3, 2627 August 1974; 3-4 pairs, 27 May-4 June 1975; c. 20, 31 May 1983 (Kirwan 1993); 3, 30 May 1986 (Schenk unpubl.); 2, 5 July 1986 (Webb unpubl.); 2, 19 August 1987 (Lister unpubl.); 1, 29 April 1988 (Rushforth unpubl.); 7, 22 May 1988 (Hodgson unpubl.); 3, 20 August 1988 (Moore unpubl.); 18, 26 April 1991 (Kirwan 1993); 89, 16 March 1994 (DHKD database); 1, 5 April 1995 (DHKD database).

Ilgaz Gölü: listed as a breeding bird without details (Kumerloeve 1961).

Kulu Gölü: records from April to November (Kasparek 1987) and current breeding population considered to be 1o pairs (M. Yarar in litt.); 5,9 July 1967 (Vielliard 1968); c. 5, 14 July 1971; 55 including 5 juveniles, 5-6 August 1972; 8, 25 August 1974; at least 10, 4 May 1979 (Kasparek 1987); 2-3, 10 May 1984 (Kasparek 1987); 1, 20 May 1984 (Harrap unpubl.); 100, 22 July 1984 (Kasparek 1987); 1, 5 May 1985 (Densley unpubl.); 200, 6 September 1986 (McLoughlin unpubl.); 2, 3 April 1988 (Schekkerman and van Roomen 1993); 10, 14 August 1988 (de Bruin unpubl.); 2, 2-5 September 1988 (Green unpubl.); 4, 13 June 1989 (Jonassohn unpubl.); 3, 18 June 1990 (Forsten unpubl.); 1, 25 July 1990 (Thewlis unpubl.); 3, 10 June 1991 (Kirwan unpubl.); 3, 29 August 1991 (DHKD database); 13, 19 June 1993 (DHKD database); 1, 16 July 1993 (Hogg unpubl.); 1, 10-11 May 1996 (Whitehouse unpubl.).

Mogan Gölü: listed as a breeding bird without details (Kumerloeve 1961), current breeding population considered to be 20 pairs (O. Görgün in litt. to DHKD but may be significantly less, (G. Eken in litt.); 2 adults, 6 July 1967 (Vielliard 1968); 5, 22 October 1967 (Vielliard 1968); 1, 4 January 1969; 3, 23 July 1972; 6, 4 August 1974; 2, 22 July 1975; 1, 20 August 1975; pair, 6 April 1985 (Novatours unpubl.); 8, 9 March 1986 (Berg unpubl.); 10, 2 April 1988 (Schekkermanand van Roomen 1993); 25, 14 May 1993 (Collin unpubl.); 200, mid-September 1994 (Welch and Welch unpubl.); less than 10, 7 July 1996 (Anatolian Duck Project 1996); 1, 23 September 1996 (Tavares unpubl.); 4, 23 December 1996 (U. Özesmi et al.). 
Palas Gölü: 1, 8 April 1988; 4, 28 April 1988; 4, 17 May 1988 (all records, Schekkerman and van Roomen 1993).

Seyfe Gölü: drake, 7 June 1981 (Husband \& Kasparek 1984); 7, 30 April 1988 (Schekkerman and van Roomen 1993).

Sultansazligi: recorded between 14 March and 7 October and current breeding population considered to be 20 pairs (Magnin and Yarar 1997) or 40 pairs (C. Bilgin in litt. to G. Eken); 5 pairs, May 1970; 2, 1 June 1975; nest, 28 April 1982; female with 6 unfledged young, 9-10 July 1982; nest, 19-22 May 1983 (all records, Kasparek 1985); 1, 29 September 1984 (Kasparek unpubl.); 15, 7 May 1988 (Ullman unpubl.); 2, 21 May 1988 (Hodgson unpubl.); 20, 4 May 1989 (Ullman unpubl.); c. 20, 22 May 1989 (Clarke unpubl.); 4, 1 June 1989 (Morton unpubl.); 3, 17 June 1989 (Jonassohn unpubl.); 2, 10 July 1990 (Bauer unpubl.); 27, 1-3 October 1990 (Khan unpubl.); 13, 7 December 1990 (Khan unpubl.); 10, 19 December 1990 (Khan unpubl.); 22, 3-4 January 1991 (Khan unpubl.); 41, 17 March 1991 (Khan unpubl.); 25, 30 March 1991 (Khan unpubl.); 17, 12 April 1991 (Khan unpubl.); 4, 26 April 1991 (Khan unpubl.); 5, 10 May 1991 (Khan unpubl.); 2, 31 May 1991 (Khan unpubl.); 8 including 3 juveniles, 1 June 1991 (Khan unpubl.); 25 including 17 juveniles, 9-10 June 1991 (Khan unpubl.); 32, 31 July 1991 (Khan unpubl.); 160, 21 August 1991 (Khan unpubl.); 75, 31 August 1991 (Khan unpubl.); 102, 12 September 1991 (Khan unpubl.); 15, 27-28 September 1991 (Khan unpubl.); 4, 18 October 1991 (Khan unpubl.); 6, 27 October 1991 (Khan unpubl.); 1, 8 November 1991 (Khan unpubl.); 1, 6 December 1991 (Khan unpubl.); 8, 18 April 1992 (Khan unpubl.); 9, 2 May 1992 (Khan unpubl.); 2, 9 May 1992 (McMahon unpub.); 30, 9 May 1992 (Petersson unpubl.); 3, 29 May 1992 (Khan unpubl.); 5, 19 June 1992 (Khan unpubl.); 3, 30 June 1992 (Khan unpubl.); 13, 29 July 1992 (Khan unpubl.); 2, 19 April 1993 (DHKD database); 10, 22 April 1993 (Collin unpubl.); 1, 14 June 1993 (DHKD database); 12, 4 June 1994 (DHKD database); 2, 21 July 1994 (van der Vliet unpubl.); 10, 21 September 1995 (Kirwan unpubl.); 13, 31 March 1996 (Royle unpubl.); less than 10, 3 August 1996 (Anatolian Duck Project 1996).

Uyuz Gölü: drake, 31 May 1991; dead female, 21 July 1991; dead female, 26 October 1991; pair, 5 April 1992; 3, 9 May 1992; 4, 2 June 1993; 6, 19 June 1993; 3 females with 5, 7 and 9 juveniles respectively, 17 and 24 July 1993 (all records, Karauz Kirac 1993).

Yarma wetlands: 3 pairs, 16 May 1971; pair, 11 June 1971.

\section{Other sites}

Hafik Gölü: recorded, 14 May 1984 (Heunk unpubl.); 4, 14 September 1994 (DHKD database).

Listed for Cimenliyeniköy, south of Tuz Gölü without details (Kumerloeve 1970).

Sariyar Baraji: 1,18 September 1995 (Kirwan unpubl.); 5, 27 April 1996 (DHKD database).

2 pairs, marsh near Esmekaya, May 1970.

4, Hirfanli Baraji, 13 February 1994 (DHKD database).

2, Kesikköprü Baraji, 13 February 1994 (DHKD database).

2, near Konya (unspecified locality), 13 April 1963 (Kumerloeve 1970).

1, Kozanli Gokgöl, 18 March 1994 (DHKD database).

8, near Temelli, 3 May 1973. 2, Temelli, 10 August 1975.

1, Todurge Gölü, 19 September 1995 (Kirwan unpubl.).

\section{South-east (o breeding sites; 2 other localities)}

Main sites (including all breeding localities)

Gölbasi Gölü west of Adiyaman: recorded, 15 July 1967 (Vielliard 1968); 3, 11 April 1982 (Gyssel unpubl.).

Kabakli Reservoir: 2, January 1993 or 1994; 1, March 1993 or 1994 (Biricik 1996).

\section{East ( 4 breeding sites; 17 other localities)}

Main sites (including all breeding localities)

Ardahan marshes ( 2 km east of village): "small numbers breed in Putka Gölü, north-east of Ardahan" (Magnin and Yarar 1997); 21, 14 September 1973; 1, 17 July 1992 (Magnin unpubl.).

Arin Gölü: 22, 17-18 September 1967 (Vielliard 1968); 3, 18 August 1972; c. 30, 21 September 1988 (Green unpubl.); 5, 13 July 1995 (DHKD database).

Bendimahi delta: 4, 26 June 1985 (Husband unpubl.); 2, 26 May 1986 (Warren unpubl.); 4, 8 July 1987 (Green unpubl.); 2, 4 June 1988 (DHKD database); at least 10, 19 September 1988 (Green unpubl.); 2, 9 May 1989 (van den Berk et al. 1993); 2 drakes, 19 May 1989 (Clarke unpubl.); 2, 23 June 1989 (Jonassohn. unpubl.); 1, 22 May 1993 (Kirwan unpubl.); 1, 8 July 1993 (Hogg unpubl.). 
Caldiran marshes: 10, 25 June 1985 (Husband unpubl.); drake, 1 June 1990 (Dubois unpubl.).

Çildir Gölü: 20, 22 September 1967 (Vielliard 1968); 6, 23 August 1973; 5, 2 September 1973; 8, 24-30 June 1988; 6, 17-23 September 1988; 12, 1-6 October 1989 (1988 and 1989 observations, Ayvaz 1991). Dogubeyazit marshes: "breeding bird" (Magnin and Yarar 1997); 2 pairs, 15 June 1973; 2, 25 May 1993 (Kirwan unpubl.).

Edremit marshes: 8 including juveniles, 29 June 1987 (Green unpubl.).

Erçek Gölü: 2, 27 May 1969; 4, 20 September 1981 (both records, Kasparek and van der Ven 1983).

Kuyucuk Gölü: 1, 21 September 1967 (Vielliard 1968); drake, 18 July 1992 (Magnin unpubl.).

Van Gölü (most records from Van town marshes): 2, late May 1969; drake, 2 July 1969; 4, 8 May 1972; "several', 18 August 1972; 1, 21 August 1972; 9, 6 September 1973; 2, 8 August 1974; c. 8, 8 June 1975; 1, 18-19 May 1975; 2, 1 June 1984 (Harrap unpubl.); c. 60, mid-May 1985 (Allport unpubl.); 2, 22 May 1985 (Cole unpubl.); 2, 23 May 1985 (Grace unpubl.); pair with 7 young, 28 \& 30 June 1985 (Husband unpubl.); 45, 24 May 1986 (Warren unpubl.); 5, 15 June 1986 (Schenk unpubl.); 2, 9 July 1986 (Webb unpubl.); 2, 14 July 1986 (Speiermann unpubl.); 9, 1 August 1986; 15, 3 August 1986; 40, 8 August 1986 (all McLoughlin unpubl.); 10, 3 June 1987 (Eames unpubl.); 4 pairs, 4 June 1987 (Hirschfeld unpubl.); 5, 5 June 1987 (Eames unpubl.); 21, 8 June 1987 (Anderson unpubl.); 45 including juveniles, 27 June-1 July 1987 (Green unpubl.); c. 80, 15 September 1988 (Green unpubl.); 2, 3 May 1989; 9, 5 May 1989; 9, 8 May 1989; 18, 11 May 1989 (all May 1989 records, van den Berk et al. 1993); 3, 19 May 1989 (Green unpubl.); 2, 24 May 1989 (Young unpubl.); at least 10, 3 I May 1989 (Smethurst unpubl.); 10, 10 June 1989 (Broome unpubl.); 10, 14 May 1990 (Ullman unpubl.); 4, 17 May 1990 (Ullman unpubl.); 1, 30 May 1990 (Dubois unpubl.); 1, 25 June 1990 (Kirwan unpubl.); 3, 4 August 1992 (Hornbuckle unpubl.); 3, 19 May 1993 (Kirwan unpubl.); 5, I June 1993 (Karlsson unpubl.); 3, 7 June 1994 (Rosier unpubl.).

\section{Other sites}

Pair, between Agri and Tasliçay, 24 May 1964 (Kumerloeve 1967).

Bulanik Ovasi: 2, 27 July 1987 (Vermeulen unpubl.); 1, 1 June 1989 (Smethurst unpubl.).

Pair, Gevas, 30 May 1990 (Dubois unpubl.). 40 adults, west of Gevas, 4 August 1981 (Helbig 1984).

c. 20, Göründï, 30 June 1987 (Green unpubl.).

4 pairs, Hazar Gölü (near Malatya), 29 July 1988 (Hofland unpubl.).

8, Nursun Gölü, north of Van Gölü, 12 July 1995 (DHKD database).

Tatvan (west end of Van Gölü): 1-2, 22 May 1968 (Kumerloeve 1969); drake, 31 May 1993 (Karlsson unpubl.).

\section{References}

Ayvaz, Y. (1991) Çildir Gölü Kuslari. Tr. J. of Zoology 15: 53-58.

Bauer, W. and Müller, G. (1969) Zur Avifauna des Evros-Delta. Beitr. Naturkdl. Forsch. SW-Deutschl. 28: 33-52.

Beaman, M. (1986) Turkey Bird Report 1976-81. Sandgrouse 8: 1-41.

Berk, V. M. van den, Cronau, J. P. and Have, T. M. van der (1993) Waterbirds in the Van province, eastern Turkey, May 1989. Zeist: WIWO Report 34.

Biricik, M. (1996) Birds of Kabakli Reservoir, Diyarbakir. Tr. J. Zool. 20: 155-160.

Collar, N. J., Crosby, M. J. and Stattersfield, A. J. (1994) Birds to watch 2: the world list of threatened birds. Cambridge, U.K.: BirdLife International (BirdLife International Conservation Series 4 ).

Dogal Hayati Koruma Dernegi (DHKD) (1992a) Towards integrated management in the Göksu delta: a protected special area in Turkey. Istanbul: DHKD Feasibility Report.

Dogal Hayati Koruma Dernegi (DHKD) (1992b) Results of the international waterfowl census, Turkey 1992. Istanbul: DHKD Bird Section report no. 6.

Dogal Hayati Koruma Dernegi (DHKD) (1996) International waterfowl census, Turkey 1996. Istanbul: DHKD Bird and Wetland Section Report no. 9.

Ertan, K. T. (1996) The birds of Kocaçay Delta. Birds of Turkey 12. Heidelberg: Kasparek Verlag. 
Have, T. M. van der, Berk, V. van den, Cronau, J. P. and Langeveld, M. (1988) South Turkey project. A survey of waders and waterfowl in the Çukurova deltas, spring 1987. Zeist: WIWO Report 22.

Helbig, A. (1984) Bemerkenswerte ornithologische Beobachtungen in der Türkei im Sommer 1981. Bonn. Zool. Beitr. 35: 57-69.

Husband, C. and Kasparek, M. (1984) The birds of Lake Seyfe. Birds of Turkey 2. Heidelberg: Kasparek Verlag.

Hustings, F. and van Dijk, A. (1994) Bird census in the Kizilirmak delta, Turkey, in spring 1992. Zeist: WIWO Report 45.

Karauz Kirac, S. (1993) The birds of Çöl Gölü. Birds of Turkey 10. Heidelberg: Kasparek Verlag.

Kasparek, M. (1985) Die Sultanssümpfe - Naturgeschichte eines Vogelparadieses in Anatolien. Heidelberg: Kasparek Verlag.

Kasparek, M. (1987) The birds of Lake Kulu. Birds of Turkey 5. Heidelberg: Kasparek Verlag.

Kasparek, M. (1988) Der Bafasee: Natur und Geschichte in der türkischen Ägäis. Heidelberg: Kasparek Verlag.

Kasparek, M. (1992) Die Vögel der Türkei: eine Übersicht. Heidelberg: Kasparek Verlag.

Kasparek, M. and van der Ven, J. (1983) The birds of Lake Erçek. Birds of Turkey 1. Heidelberg: Kasparek Verlag.

Kiliç, A. and Kasparek, M. (1987) The birds of Lake Yeniçaga. Birds of Turkey 6. Heidelberg: Kasparek Verlag.

Kiliç, A. and Kasparek, M. (1989) The birds of the Köycegiz-Dalyan area. Birds of Turkey 8. Heidelberg: Kasparek Verlag.

Kiliç, A. and Kasparek, M. (1990) The Eregli Marshes (Turkey): assessment of their biological importance and recommendations for conservation. Cambridge U.K.: International Council for Bird Preservation/World Wildlife Fund.

Kirwan, G. (1990) The wetlands at Eregli, Turkey. Orn. Soc. Middle East Bull. 25: 23-25.

Kirwan, G. (1993) The birds of the Hotamis Marshes. Birds of Turkey 9. Heidelberg: Kasparek Verlag.

Kivit, H., Nijmeijer, H. and Ovaa, A. (1994) Wader and waterfowl migration in the Çukurova deltas, south Turkey, spring 1990. Zeist: WIWO Report 48.

Kiziroglu, I., and Kiziroglu, F. (1987) Die Vogelarten im Vogelparadies des Kus Cenneti/ Bandirma Nationalparks und seiner Umbegung. Verh. Orn. Ges. Bayern 24: 515-532.

Kumerloeve, H. (1961) Zur Kenntnis der Avifauna Kleinasiens. Bonn. Zool. Beitr., Sonderheft.

Kumerloeve, H. (1963) L'avifaune du Lac d'Äntioche (Amik Gölü - Göl Basi) et de ses alentours. Alauda 31: 161-211.

Kumerloeve, H. (1966) Migration et hivernage sur le Lac d'Antioche (Amik Gölü, Hatay, Turquie); coup d'oeil sur son avifaune nidificatrice actuelle. Alauda 34: 299-308.

Kumerloeve, H. (1967) Neue Beiträge zur Kenntnis der Avifauna Nordost und OstKleinasiens. Istanbul Üniv. Fen. Fak. Mecm. 32: 79-213.

Kumerloeve, H. (1969) Zur Avifauna des Van Gölü - und Hakkârigebietes (E/SE Kleinasien). Istanbul Üniv. Fen. Fak. Mecm. 34: 245-312.

Kumerloeve, H. (1970) Zur Kenntnis der Avifauna Kleinasiens und der europäischen Türkei. Istanbul Üniv. Fen. Fak. Mecm. 35: 85-16o.

Langeveld, M. and Ruiters, P. S. (1994) Breeding bird census at Eber Gölü, Aksehir Gölü and Karamik Gölï 1994. Istanbul: DHKD Bird \& Wetland Section Report 8.

Magnin, G. and Yarar, M. (1997) Important bird areas of Turkey. Istanbul: DHKD.

Martins, R. P. (1989) Turkey Bird Report 1982-86. Sandgrouse 11: 1-41.

Mathey-Dupraz, A. (1924) Notes ornithologiques de la région de Bosphore. Orn. Beob. 22: 6-8. 
Ornithological Society of Turkey (OST) (eds.) (1969) Bird Report 1966-1967. Sandy, U.K.: OST.

Ornithological Society of Turkey (OST) (eds.) (1972) Bird Report 1968-1969. Sandy, U.K.: OST.

Ornithological Society of Turkey (OST) (eds.) (1975) Bird Report 1970-1973. Sandy, U.K.: OST.

Ornithological Society of Turkey (OST) (eds.) (1978) Bird Report 1974-1975. Sandy, U.K.: OST.

Schekkerman, H. and van Roomen, M. W. J. (1993) Migration of waterbirds through wetlands in central Anatolia. Zeist: WIWO Report 32.

Tucker, G. M. and Heath, M. F. (1994) Birds in Europe: their conservation status. Cambridge, U.K.: BirdLife International (BirdLife Conservation Series 3).

Vauk, G. (1973) Ergebnisse einer ornithologischen Arbeitsreise an den Beysehir Gölü (SW-Anatolien) im April/Mai 1964. Beitr. Vogelkde. 19: 225-26o.

Vielliard, J. (1968) Türkiyede bir ornitolojik gezinin neticleri. Istanbul Üniv. Fen. Fak. Mecm. 33: 67-170.

Warncke, K. (1965) Beitrag zur Vogelwelt der Türkei. Vogelwelt 86: 1-19.

van Winden, A., Mostert, K. van, Ruiters, P., Siki, M. \& Waard, H. de (1989) Waders and waterfowl in spring 1988 at Eber Gölü. Zeist: WIWO Report 28.

GUY M. KIRWAN

6 Connaught Road, Norwich NR2 3 BP, U.K. 